\title{
Christoph Neuberger:
}

\section{Funktionen, Probleme und Regulierung von Suchmaschinen im I nternet}

\begin{abstract}
English in the article
Suchmaschinen haben eine Orientierungs- und Speicherfunktion im Internet. Der Wettbewerb zwischen Google, Yahoo und Microsoft, der im Jahr 2004 an Schärfe gewonnen hat, wird als „Krieg der Architekturen“ interpretiert, bei dem es letztlich darum geht, allgemeine Standards für die Aufbereitung und Suche digitaler Informationen zu setzen. Die Frage, wie groß der Einfluss des Marktführers "Google“ auf die Aufmerksamkeitslenkung im Internet ist, lässt sich noch nicht abschließend beantworten. Gegen ein "Googlepol“ spricht zum Beispiel, dass viele Nutzer parallel auch bei anderen Anbietern suchen. Die Qualität der SuchmaschinenErgebnisse wird nicht nur durch technische Schwächen, sondern in wachsendem Maße auch durch externe und interne Formen der Manipulation beeinträchtigt. In der letzten Zeit haben sich Suchmaschinen-Betreiber und Suchmaschinen-Optimierer in Selbstverpflichtungserklärungen auf Regeln geeinigt, durch die mehr Transparenz für die Nutzer geschaffen und das Problem des "Spamming" von Suchmaschinen gelöst werden soll.
\end{abstract}

\section{Agenda}

Extended Abstract: Function, Problems, and Regulation of Search Engines in the Internet ..................... 4

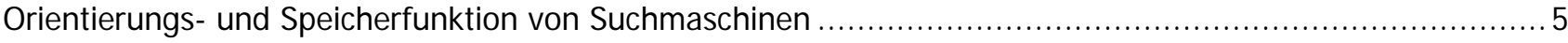

Die großen Drei auf dem globalen Suchmaschinen-Markt: Google, MSN und Yahoo ............................... 6

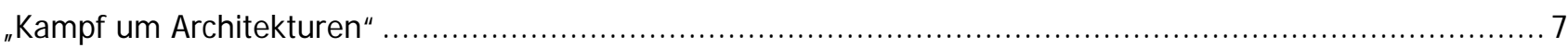

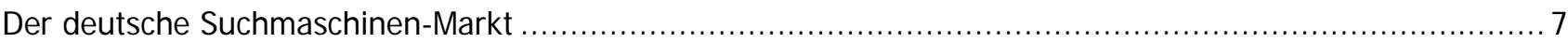

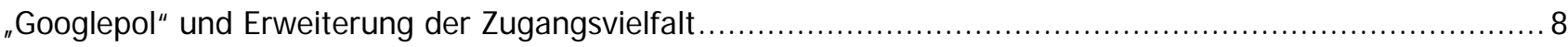

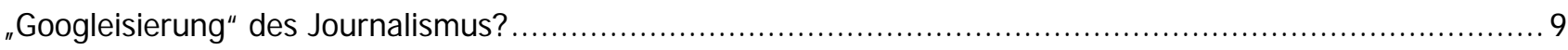

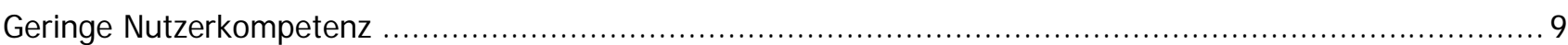

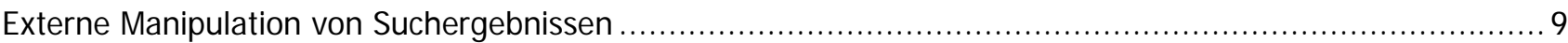

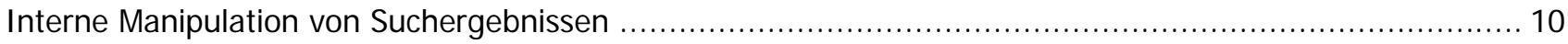

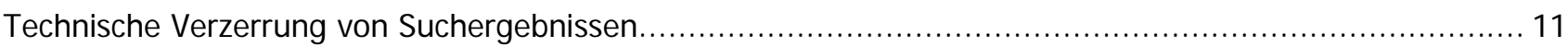

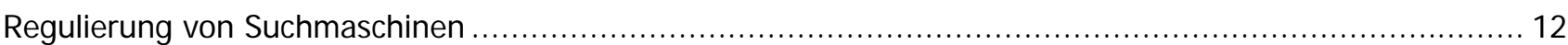

Author:

Prof. Dr. Christoph Neuberger:

- Organization and contact address: Institut für Kommunikationswissenschaft (IfK) Westfälische Wilhelms-Universität Münster, Bispinghof 9-14, 48143 Münster, Germany

- Telephone, email and personal homepage: $\mathbf{m}+49$ - 251-8323002, $\square$ neuberger@uni-muenster.de 믄.

- Relevant publications: See: http://egora.uni-muenster.de/ifk/personen/neubergerpublikationen.htm 


\section{Extended Abstract: Function, Problems, and Regulation of Search Engines in the Internet}

Search engines are the most used type of offer in the internet (van Eimeren/Gerhard/Frees 2003: 35). However, they have hardly been analyzed yet in communications sciences. Most of all, search engines have an orientation function but at the moment are also increasingly significant as stores of information. Thus, the market leader Google in 2004 made an agreement with five big libraries on digitalizing 15 millions of books and documents. Particularly in France this announcement provoked reaction, as they said that a cultural inequality was to be feared if predominantly literature in the English language was made accessible in this way.

Altogether, the competition of the three great suppliers Google, Yahoo, and Microsoft has increased during the year 2004. Google going public on August 19th, 2004, drew attention at the search engines market. The company made returns of 1.67 Billions of US-Dollars, more than 500 of which were supposed to be invested in the extension of the search engine in 2005. In the course of the year the two main rivals Yahoo and Microsoft caught up: Yahoo split up with Google and invested 2 Billions of US-Dollars. Microsoft gained independence from Google and in November, 2004, started the testversion of its own search-technology. After the competitors having caught up with most of Google's lead, experts since recently consider the possibilities of search-technology to be exhausted. Thus, competition has shifted towards specialized search offers and supplementary services. Altogether, functional expansion and convergent development are to be observed, the great suppliers thus coming closer to each other.

According to Ferguson's opinion (2005: 39), competition develops towards a "war of architectures": Finally, he says, it is all about defining standards for processing and search of digital information on all technological platforms. De facto, standards are set up by the market leader, because of which the competing entrepreneurs will at first try to reach a share of the market which is as big as possible.

The competition of the great US-search engines also influences the German market, where the "global players" appear with country-specific offers. For 2004, a total of 193 offers in the German language were investigated. In 2002, one third of the German-speaking search engines were run without commercial interest, one fifth served the image of a company. Almost one half was part of a portal (cf. Machill/Welp 2003: 76f.).

The question of how big the market leader Google's influence is on controlling attention in the internet cannot yet be finally answered. Basically, however, there are limits for controlling the attention of search engines: while traditional mass media decide about which news and opinions are published, search engines only inform about already existing offers. Moreover, a growing share of users is not interested any more in new sites (cf. van Eimeren/Gerhard/Frees 2003: 354f.), thus trends indicate that the demand for search-performance may decrease. The majority of users also searches parallel in one of the two other top-3 search engines (cf. Nielsen NetRatings 2005). Google's influence on traditional mass media, the so called "Googleization" (Seifert 2003), has not yet been confirmed by studies.

Tests show that despite the huge market share the quality of Google has not discernibly worsened in comparison to other search engines (cf. Neuberger 2005: 6f.). A part of the users critical attitude towards business leads to the operators being closely watched and irregularities being published (e. g. at google-watch.org or in Germany by "Telepolis").

Several studies give evidence to the low degree of competence of search engine users both regarding the evaluation of hit-lists and working the search engines (cf. Machill/Welp 2003: 166-175, 341-345; eprofessional 2004). The different competence of the users might result in a digital divide of the second order (cf. Marr 2005: 28).

The quality of search engines is not only affected by technological weak spots but increasingly also by external and internal ways of manipulating. Results are externally manipulated by search engine optimizers who on behalf of suppliers are supposed to improve the chances of attention of certain websites. Most of all, affiliate-programmes by onlineshops and auctions have contributed to an increasing share of spam at Google (cf. Karzauninkat 2004b: 90; Roush 2005). In the course of a survey among German-speaking suppliers of search engines it occurred that rather easily applicable kinds of spamming are predominating, , e.g. using false keywords within the meta-tags (cf. Machill/Welp 2003). 
The problem of internal manipulation is in hits which are paid for by website suppliers and which are insufficiently or not at all labelled as being commercial. In 2001 the consumer advocates' association Commercial Alert pointed out to this practice by filing a suit at the Federal Trade Commission (FTC). Also search engines in the German language combine commercial banners to searches regarding subject matter; payed results are also widely spread (cf. Machill/Welp 2003: 90f.). Most of all, bought places on the hit lists themselves seem to be a problem. Studies show that many users do not know the practice of payed hits (cf. Princeton Survey Research Associates 2002: 17; Machill/Welp 2003: 179f.; Marable 2003; Frankfurter Allgemeine Zeitung 2005). Informed online-users in their majority demanded clear labelling. Of these, 45 per cent also stated that they would stop using a search engine if paid hits were not appropriately labelled (cf. Fallows 2005: 16-21)

Obviously, Google differentiates the accessibility of certain sites according to countries. Due to this, this search engine was confronted by the accusation of intransparent influence on search results and of over-hastily giving way to political pressure (cf. e. g. Palm 2002; Zittrain/Edelman 2002; Finkelstein 2003; Jodda 2003; McHugh 2003; Rötzer 2004; Schwan 2004c). On the other hand, Google and other search engines are also criticized for making access to problematic sites (pornography, propaganda etc.) possible.

In Germany, during the past few years there have been intensive debates on (self)regulation of search engines. A first try was made by the Bertelsmann Stiftung (Bertelsmann Foundation) which in 2003 introduced a code of conduct regarding the selfobligation of search engine-suppliers. However, response was very low, so that further steps (seal of quality, founding an organization of self-control) were given up (cf. Bertelsmann Stiftung 2003, 2004). In February, 2005, the "Freiwillige Selbstkontrolle Multimedia-Anbieter" (FSM) (Self-Control Organization of MultiMedia Suppliers) introduced a code of conduct for search engines (cf. FSM 2004). It also includes a procedure of complaints and a list of sanctions. Moreover, the search engines obliged themselves not to make accessible those sites as put on the index by the Bundesprüfstelle für jugendgefährdende Medien (BPjM) (Federal Review Board for Media Harmful to Young Persons). Such detailed indications are lacking in the case of foreign suppliers, however. The Bundesverband Digitale Wirtschaft (BVDW) (Federal Association of Digital Business) decided in 2004 to grant in future a certificate to respectable marketing agencies for search engines in order of checking the spamming of search engines. However, it is much too early to draw a conclusion regarding the effect of these kinds of self-control.

\section{Orientierungs- und Speicherfunktion von Suchmaschinen}

Suchmaschinen sind in der Kommunikationswissenschaft bisher noch kaum analysiert worden, ${ }^{1}$ obwohl sie der meistbesuchte Angebotstyp im Internet sind (vgl. van Eimeren/Gerhard/Frees 2003: 35). Der Grund für die große Bedeutung von Metamedien im Internet sind die spezifischen Orientierungsprobleme und -potenziale des Internets. Das Internet vereinfacht den Zugang zur Öffentlichkeit: Kommunikatoren sind nicht mehr auf Redaktionen angewiesen, die als "Gatekeeper" Aussagen prüfen und selektieren, sondern können selbst als Anbieter auftreten. Die Nutzer ihrerseits haben einen unvermittelten Zugriff auf eine Vielzahl von Angeboten. Dadurch sind sie aber auch alleine mit der Aufgabe konfrontiert, aus der Überfülle verfügbarer Seiten eine sinnvolle Auswahl zu treffen. Aus der Sicht der Anbieter schwindet angesichts der "Informationsflut" die Wahrscheinlichkeit, die Aufmerksamkeit von Nutzern zu gewinnen und Anschlusskommunikation auszulösen.

Nach der ARD/ZDF-Online-Studie 2004 sind für drei Viertel der Online-Nutzer (74 \%) Suchmaschinen die zentrale Quelle für das Auffinden neuer Seiten (vgl. van Eimeren/Gerhard/Frees 2004: 355). Sie sind damit die wichtigsten Navigatoren, die den Weg zu Informationen im Internet weisen. Digital aufberei-

1 Im Rahmen des Forschungsprojekts "Transparenz im Netz" (2002/2003) wurden erstmals umfassend die Anbieter, Angebote und Nutzer deutschsprachiger Suchmaschinen empirisch analysiert. Die Bertelsmann Stiftung hat dafür Forscher an den Universitäten Münster und München beauftragt. Dabei wurden eine Anbieterbefragung, Inhaltsanalysen von Trefferlisten (Christoph Neuberger), eine repräsentative Nutzerbefragung (Wolfgang Schweiger), Laborexperimente (Werner Wirth) sowie ein Test von Seiten-Optimierungsverfahren (Christoph Neuberger, Stefan Karzauninkat) durchgeführt. Die in diesem Beitrag zitierten Projektergebnisse sind im Sammelband von Machill/Welp (2003) dokumentiert. 
tete Informationen haben den Vorteil, dass Suchmaschinen in kurzer Zeit große Mengen davon durchforsten und relevante Seiten anzeigen können, die bestimmte Suchwörter enthalten. Mit Hilfe eines "Crawler" oder "Spider" genannten Agenten erfassen sie große Teile des Internets und indexieren die in den Dokumenten auftauchenden Wörter.

Darüber hinaus gewinnen Suchmaschinen-Anbieter als Informationsspeicher an Bedeutung. Der Marktführer Google gab im Dezember 2004 bekannt, dass das Unternehmen eine Vereinbarung mit fünf renommierten Bibliotheken über die Digitalisierung von 15 Millionen Büchern und Dokumenten geschlossen hat. Beteiligt sind die Universitäten Stanford, Harvard, Michigan und Oxford sowie die New York Public Library. Das Projekt ist auf zehn Jahre angelegt. Die auf mindestens 150 Millionen USDollar geschätzten Kosten werden alleine von Google getragen. Ältere Bücher werden vollständig über das Internet verfügbar gemacht, urheberrechtlich geschützte Werke in Auszügen (vgl. Krüger 2004a; Lee 2004). Die Ankündigung von Google, Bibliotheksbestände zu digitalisieren, hat vor allem in Frankreich Reaktionen ausgelöst: Der Leiter der Nationalbibliothek, Jean-Noël Jeanneney, sah die Gefahr eines kulturellen Ungleichgewichts im Internet, wenn in erster Linie englischsprachige Literatur im weltweiten Netz öffentlich zugänglich wird, und forderte ein europäisches Gegenprojekt. Staatspräsident Jacques Chirac will der Europäischen Union die Digitalisierung der Werke der großen europäischen Bibliotheken vorschlagen (vgl. Wiegandt 2004: 15; Neue Zürcher Zeitung 2005c: 51).

\section{Die großen Drei auf dem globalen Suchmaschinen-Markt: Google, MSN und Yahoo}

Im Jahr 2004 ist der Suchmaschinen-Markt in Bewegung geraten. Vor allem der Börsengang der Suchmaschine Google am 19. August hat die öffentliche Aufmerksamkeit auf den Markt der Suchmaschinen gelenkt. Google erzielte einen Erlös von 1,67 Milliarden US-Dollar, der in den weiteren Ausbau der Suchmaschine fließen soll. Allein im Jahr 2005 sollen über 500 Millionen US-Dollar investiert werden (vgl. Bauer 2004: 23; Virtel 2005). Im Laufe des Jahres 2004 holten die beiden Hauptrivalen Yahoo und Microsoft gegenüber Google auf: Yahoo hat im Februar 2004 seine Kooperation mit Google beendet und arbeitet seither mit einer eigenen Suchtechnik. Yahoo hat 2 Milliarden US-Dollar investiert und unter anderem den Suchdienstleister Inktomi und die
Vermarktungsfirma Overture übernommen. Microsoft machte sich unabhängig von den Ergebniszulieferungen von Yahoo. Im November 2004 startete MSN die Testversion einer technisch eigenständigen Suchmaschine (vgl. Bager 2004b; Fischermann 2004; Hohensee 2004; Laube 2004; Patalong 2004a; Ferguson 2005: 36-42).

Nachdem die Konkurrenten den Vorsprung von Google weitgehend aufgeholt haben, erscheint Experten die Suchtechnik derzeit ausgereizt. Mit großen Technologiesprüngen ist nicht zu rechnen. Unterschieden wird bei der Suchtechnik zwischen herkömmlichen Gewichtungsmodellen, bei denen die Häufigkeit der Suchwörter in den Dokumenten bestimmt wird, und neueren Modellen, bei denen die Hypertextstruktur, in die ein Dokument eingebettet ist (wie beim „Page Rank“-Verfahren von Google), oder die Nutzungshäufigkeit von Seiten ("Click Popularity“) ausgewertet werden (vgl. Biever 2004; Glöggler 2003: 67-94).

Der Wettbewerb hat sich auf spezialisierte Suchangebote sowie Zusatzdienste verlagert, die den Nutzern kostenlos offeriert werden, um Marktanteile zu gewinnen oder zu sichern. Google hat seit der Ankündigung des Börsengangs eine Vielzahl neuer Angebote gestartet (Google-Funktionen: www. google.de/intl/de/features.html). Über eine deutschsprachige Nachrichtensuche verfügen inzwischen neben Google auch Yahoo und Web.de. Ein weiteres Expansionsfeld ist die lokale Suche: Yahoo Deutschland plant für das erste Quartal 2005 ein solches Angebot und kooperiert dabei mit dem Telefonbuchanbieter "Das Örtliche“, der zum Telekom-Konzern gehört; Google will noch im Laufe des Jahres damit starten (vgl. Golem 2004; sueddeutsche.de 2005). Seit Januar 2005 können in den USA Fernsehprogramme über "Google Video“ recherchiert werden; Yahoo kündigte an, bald nachzuziehen (vgl. Spiegel Online 2005). Von den großen Anbietern wird auBerdem die Entwicklung von Video-Suchmaschinen vorangetrieben; Yahoo hat im Dezember 2004 eine Videosuche vorgestellt (vgl. Olsen/Kaufman 2004; Neue Zürcher Zeitung 2005d).

Neben neuen Suchofferten hat Google im April 2004 mit G-Mail auch einen Mailservice gestartet. Damit hat Google sein Leistungsspektrum über die Suchfunktion hinaus auch auf Kommunikationsangebote erweitert und greift damit seine schärfsten Konkurrenten Yahoo und MSN auf einem wichtigen Feld an. G-Mail geriet aber ins Visier von Datenschützern, weil zum Inhalt der Mails passende Werbung geschaltet werden soll (vgl. Bleich/Heidrich 2004; Patalong 2004b; Spiegel Online 2004). Ähnliche 
Kritik erntete Google mit seiner Desktop-Recherche, die seit Oktober 2004 in einer Testversion kostenlos heruntergeladen werden kann. Voraussetzung für eine Suche auf der eigenen Festplatte ist nämlich, dass deren gesamter Inhalt indexiert wird, wodurch die Nutzeraktivitäten nachvollziehbar werden. Auch Yahoo sucht seit Januar 2005 auf dem Desktop (vgl. Krüger 2004b; Neue Zürcher Zeitung 2005a).

Google hat darüber hinaus Publikationsmöglichkeiten für Online-Nutzer geschaffen. Dazu gehört der kostenlose Weblog-Dienst Blogger.com, der seit kurzer Zeit auch in deutscher Sprache angeboten wird (vgl. Schwan 2004a). Außerdem übernahm Google im Juli 2004 die Website Hello, auf der Nutzer ohne Gebühr Online-Fotoarchive anlegen können (vgl. Neue Zürcher Zeitung 2004). Schließlich startete im April 2005 das "Google Video Upload Program“, eine Präsentationsplattform für Hobbyfilmer (vgl. Focus Online 2005).

Insgesamt sind also eine funktionale Expansion ( $\mathrm{vgl}$. Khopkar et al. 2003) und konvergente Entwicklung zu beobachten, wodurch die großen Anbieter einander näher rücken: Während die beiden Portale Yahoo und MSN ihre Suchkompetenz verbessert haben, verbreitert die ehemals reine Suchmaschine Google inre Angebotspalette.

\section{„Kampf um Architekturen“}

Nach Auffassung von Charles $\mathrm{H}$. Ferguson steuert die Konkurrenz zwischen Google, Microsoft und Yahoo auf einen "Krieg um Architekturen“ (Ferguson 2005: 39) zu: Letztlich ginge es darum, Standards für die Aufbereitung und Suche digitaler Informationen auf allen technischen Plattformen zu definieren. Derzeit sei das "Suchuniversum ein heilloses Durcheinander, voller unerschlossener und gegeneinander abgeschotteter Bereiche. Eine gemeinsame Architektur könnte das ändern." (ebd.: 44) De factoStandards werden durch den Marktführer gesetzt, weshalb die konkurrierenden Unternehmen zunächst versuchen, einen möglichst hohen Marktanteil zu erzielen.

"Siegreiche Architekturen sind proprietär und schwer nachzubauen, aber sie sind auch offen in dem Sinne, dass sie Schnittstellen zur Verfügung stellen, auf deren Grundlage andere Anbieter und die Nutzer selbst die verschiedensten Anwendungen entwickeln können. Auf diese Weise kann eine Architektur alle Märkte erreichen, und es entsteht ein ,Lock in'-Effekt: Die Nutzer werden darin gefangen, weil sie nur mit großer Mühe und zu hohen Kosten zu einem anderen System wechseln können." (ebd.: 40)

Google mangelt es bislang noch an solchen Schnittstellen, wie sie z.B. Microsoft für das Betriebsprogramm Windows bereitstellt, um für fremde Programme anschlussfähig zu sein. Wer auch immer die Standards setzen wird: Das siegreiche Unternehmen würde einen vereinheitlichten Suchmarkt kontrollieren.

„Einer der besten Gründe, auf das Überleben von Google zu hoffen, ist (...), dass bessere Qualität zu erwarten ist, wenn der Wettbewerb hart bleibt. Wenn Google die Suchindustrie dominieren würde, bliebe immer noch Microsoft als disziplinierender Faktor. Wenn dagegen Microsoft alles dominieren würde, hätten wir noch weniger Schutz vor seiner Mittelmäßigkeit. " (ebd.: 47)

\section{Der deutsche Suchmaschinen- Markt}

Der Wettbewerb zwischen den großen Suchmaschinen mit Sitz in den USA beeinflusst auch den deutschen Suchmaschinen-Markt. Die "Global Players" sind auch hier mit länderspezifischen Angeboten vertreten. Daneben umfasst der nationale Markt eine Vielzahl weiterer Suchmaschinen: Im September 2004 ließen sich 193 deutschsprachige Angebote ermitteln, bei denen die externe Suche die zentrale Funktion war und die thematisch nicht spezialisiert waren (eigene Erhebung; Auswertung einschlägiger Linkverzeichnisse). Allerdings ist die Nutzung der meisten dieser Suchmaschinen marginal im Vergleich zu den Branchenriesen. Ein Drittel der deutschsprachigen Suchmaschinen wurde 2002, so ergab einer Befragung, ohne kommerzielles Interesse betrieben, ein Fünftel diente der Selbstdarstellung eines Unternehmens. Knapp die Hälfte der Suchmaschinen war Teil eines Portals mit zahlreichen anderen Angeboten (vgl. Machill/Welp 2003: 76f.). Die reichweitenstärksten Suchmaschinen aus Deutschland sind Lycos, Fireball, Web.de und TOnline (vgl. ebd.: 156-162). Mit Seekport startete im Dezember 2003 eine neue Suchmaschine mit großen Ambitionen, die auch bereits ins europäische Ausland expandiert ist (vgl. Computerwoche 2005). 


\section{"Googlepol“ und Erweiterung der Zugangsvielfalt}

Besitzt Google ein Quasi-Monopol auf dem Suchmaschinen-Markt und damit einen großen Einfluss auf die Aufmerksamkeitslenkung im Internet? Ist die Rede vom "Googlepol“ (Winterbauer 2003) berechtigt? Vorgetragen wurde diese Sorge vor allem von der Bertelsmann Stiftung, deren Vertreter behaupten, dass Google einen Marktanteil von 70 Prozent erreiche, „und das ist eine Monopolstellung, die weder im Printbereich noch bei den elektronischen Medien zugelassen werden würde." (Machill 2004)

Für eine Berechnung von Marktanteilen mangelt es allerdings an einer soliden Datengrundlage. Die vorliegenden Statistiken kommen je nach Indikator zu sehr unterschiedlichen Ergebnissen (vgl. Machill/Welp 2003: 156-162; Neuberger 2005: 5f.). Nielsen NetRatings ermittelte für die USA im Januar 2005, dass 47 Prozent der Suchanfragen („Searches") auf Google entfielen, 21 Prozent auf Yahoo und 13 Prozent auf MSN (vgl. Nielsen NetRatings 2005). Berücksichtigt werden muss bei Marktanteilsberechnungen auch die Zulieferung von Treffern an andere Anbieter. Die Suchmaschinen sind - für die Nutzer kaum durchschaubar - untereinander eng verflochten. Der Informationsdienst "Search Engine Watch" stellte im Juli 2004 folgende Kooperationen fest: Google lieferte Treffer an AOL, Excite, Ask Jeeves, HotBot, Lycos, Netscape und Teoma. Yahoo/Overture gaben an MSN, AltaVista, AllTheWeb, HotBot und Lycos Ergebnisse weiter (vgl. Sullivan 2004; Karzauninkat 2004a). MSN stützt sich - wie erwähnt - seit November 2004 auf eine eigene Suchtechnik und bezieht keine Treffer mehr von Yahoo.

Grundsätzlich sind der Aufmerksamkeitslenkung von Suchmaschinen jedoch enge Grenzen gesetzt: I hre "Gatekeeper"-Funktion ist nicht mit jener der Redaktionen von Presse und Rundfunk vergleichbar. Traditionelle Massenmedien entscheiden darüber, welche Nachrichten und Meinungen publiziert, also dem Publikum überhaupt zugänglich gemacht werden. Suchmaschinen orientieren lediglich über Angebote, die für die Nutzer technisch bereits verfügbar sind und die sie auch auf Alternativwegen erreichen könnten. Suchmaschinen werden außerdem nur gebraucht, wenn neue Angebote gesucht werden bzw. die bekannten Angebote ein Bedürfnis nicht befriedigen können. Nicht die gesamte Internetnutzung ist deshalb von Suchmaschinen abhängig.
Die ARD/ZDF-Online-Studie zeigt, dass ein wachsender Anteil von Nutzern kein großes Interesse mehr an neuen Seiten hat und sich die Zahl der durchschnittlich pro Onlinesitzung besuchten Websites verringert (vgl. van Eimeren/Gerhard/Frees 2003: 354f.), damit dürfte tendenziell auch der Bedarf an Suchleistungen sinken. Die Abhängigkeit von Google hält sich auch deshalb in Grenzen, weil eine Mehrheit der Google-Nutzer parallel auch in einer der beiden anderen Top 3-Suchmaschinen (Yahoo, MSN) sucht, wie eine Erhebung von Nielsen NetRatings (2005) in den USA im J anuar 2005 ergab.

Tests zeigen, dass sich die Qualität von Google trotz des großen Marktanteils im Vergleich zu den anderen Suchmaschinen nicht erkennbar verschlechtert hat (vgl. Neuberger 2005: 6f.). Der wachsende Anteil gespamter Seiten in den Ergebnislisten ist darauf zurückzuführen, dass Google das wichtigste Ziel von Suchmaschinenoptimierern ist (vgl. Karzauninkat 2004b). Auch die Zufriedenheit der Onlinenutzer mit dem Marktführer ist hoch: Wolfgang Schweiger stellte in einer repräsentativen Befragung fest, dass Nutzer, die ihre Hauptsuchmaschine bewerten sollten, im Fall von Google die besten Noten vergaben (vgl. Machill/Welp 2003: 176f.). Ihre Urteilsfähigkeit dafür war auch relativ groß: „Google-Nutzer stellen die Gruppe mit der höchsten Internet- und Suchmaschinenkompetenz dar, sie nutzen am stärksten das Internet und Suchmaschinen (...).“ (ebd.: 164) Eine hohe Zufriedenheit mit Suchmaschinen im Allgemeinen und mit Google im Besonderen ergab auch eine repräsentative Nutzerbefragung in den USA im Jahr 2004 (vgl. Fallows/Rainie 2004: 3; Fallows 2005: 8-11).

Auf dem Suchmaschinen-Markt ist die Position von Google nicht zementiert. Wie gezeigt, muss Google derzeit große Anstrengungen unternehmen, um seine Position gegenüber MSN und Yahoo zu behaupten. Zwar sind die Eintrittsbarrieren für neue Wettbewerber hoch, soweit es um universelle Suchangebote für das Internet geht. Kleinen Start upUnternehmen gelingt es jedoch immer wieder, mit spezifischen Suchfunktionen erfolgreich zu sein. Erst allmählich werden das Tiefenweb und andere Plattformen wie der PC von Suchmaschinen erschlossen (vgl. Ferguson 2005: 42-44).

Die unternehmenskritische Haltung eines Teils der Nutzerschaft im Internet führt dazu, dass die Betreiber großer kommerzieller Websites unter genauer Beobachtung stehen und Missstände öffentlich deutlich zur Sprache gebracht werden. Dies geschieht im Fall von Google zum Beispiel auf der Website "Google Watch" (google-watch.org) oder im 
deutschsprachigen Onlinemagazin "Telepolis" (telepolis.de). In Deutschland wurde im Juli 2004 der gemeinnützige "Verein zur Förderung der Suchmaschinen-Technologie und des freien Wissenszugangs" (suma-ev.de) gegründet, der gegen die Konzentration auf dem Suchmaschinen-Markt kämpfen will. Als Alternative zu den kommerziellen Suchmaschinen soll in Deutschland das Entstehen eines Netzwerks aus kleinen Suchmaschinen gefördert werden, das auf "Open Source"-Software basiert (vgl. Heise Online 2004b; Sander-Beuer 2005). Um den Wettbewerb zu fördern und die Vielfalt der Perspektiven zu erweitern, wird derzeit die "Open Source"-Software Nutch entwickelt, die es jedem interessierten Nutzer erlauben soll, eine eigene Suchmaschine zu betreiben (vgl. Krempl 2004a).

\section{„Googleisierung“ des Journalismus?}

Der Einfluss von Google soll auch durch traditionelle Massenmedien verstärkt werden, weil sich Journalisten angeblich zunehmend mit Google-Anfragen zufrieden geben und auf Offline-Recherchen verzichten. Dieses als "Googleisierung" (Siegfried Weischenberg, zitiert nach Seifert 2003) des Journalismus bezeichnete Phänomen ist allerdings noch nicht durch empirische Studien bestätigt. Der Wissenschaftsredakteur des Nachrichtenmagazins „Focus", J ochen Wegner (2005), vermutet folgenden Umgang mit Google in den Redaktionen: Die Benutzung von Google definiere mittlerweile den Mindeststandard der journalistischen Recherche, was angesichts der geringen durchschnittlichen Rechercheleistung in deutschen Redaktionen "das allgemeine Niveau nur gehoben haben" könne. Google könne dazu beitragen, einfache Fehler schnell aufzuklären. Allerdings verführe Google auch dazu, sich nur auf die über die Suchmaschine erreichbaren Quellen zu beschränken. Dies aber sei gefährlich, da die von Google entdeckten Seiten im Internet Themen und Meinungen oft verzerrt widerspiegeln. So sei die Trefferzahl in Google keineswegs ein Indikator für die Wichtigkeit eines Sachverhalts oder einer Person. Schließlich bestehe auch die Gefahr, dass Falschinformationen aus dem Internet gefischt und über Massenmedien weiter verbreitet werden.

\section{Geringe Nutzerkompetenz}

Mehrere Studien belegen eine geringe Kompetenz der Suchmaschinen-Nutzer: Trefferlisten werten sie nur oberflächlich aus, sie begnügen sich meistens mit der Auswertung der ersten Ergebnisseite und der Prüfung weniger Treffer. Auch ihre Kompetenz zur Bedienung der Suchmaschinen ist wenig entwickelt (vgl Machill/Welp 2003: 166-175; 341-345; eprofessional 2004). Die unterschiedliche Kompetenz bei der Bedienung von Suchmaschinen könnte eine „digitale Spaltung“ zweiter Ordnung zur Folge haben, weil die Nutzer in sehr unterschiedlichem Maße vom Angebot des Internets Gebrauch machen können (vgl. Marr 2005: 28). Um dies zu vermeiden, müssten Suchmaschinen benutzerfreundlicher gestaltet werden. Unterstützt wird dieses Bemühen auch durch Informationsdienste wie die "Suchfibel" (suchfibel.de) oder "Search Engine Watch“ (searchenginewatch.com), die zum Verständnis der Funktionsweise von Suchmaschinen beitragen und einen Marktüberblick geben.

\section{Externe Manipulation von Suchergebnissen}

Suchmaschinen sind keineswegs die neutralen und technisch perfekten Wegweiser im Internet, als die sie erscheinen mögen (vgl. Winkler 2002: 34). Neben Schwächen der Suchtechnik beeinträchtigen in wachsendem Maße Formen der externen und internen Manipulation die Qualität ihrer Ergebnisse. Dabei erhalten Seiten höhere Ränge in Trefferlisten zugewiesen, als ihnen nach ihrer Bedeutung für die Beantwortung der Suchanfrage zustehen würden. Knapp 60 Prozent der deutschen Onlinenutzer beklagen den hohen Anteil an „Infomüll“, der auf den Trefferlisten von Suchmaschinen zu finden ist, ergab eine repräsentative Forsa-Umfrage im Jahr 2004 (vgl. Seekport 2004).

Extern manipuliert werden Ergebnisse durch Suchmaschinen-Optimierer, die im Auftrag von Anbietern die Beachtungschancen von Websites verbessern sollen. Optimierern ist es auch längst gelungen, das anfangs als kaum manipulierbar geltende „Page Rank"-Verfahren von Google, bei dem nicht der Inhalt des Dokuments, sondern dessen Vernetzungsstruktur ausgewertet wird, durch so genannte „Linkfarmen“ zu beeinflussen. Dabei wird durch Tausende untereinander verlinkter Seiten mit den passenden Stichwörtern ein optimales Umfeld für jene Seiten geschaffen, die in Google-Ergebnislisten einen hohen Rang erzielen sollen.

Vor allem Affiliate-Programme von Onlineshops und Auktionen wie Amazon und eBay, bei denen Provisionen an Websitebetreiber bezahlt werden, die Kunden zuführen, haben dazu beigetragen, dass bei 
Google der Spam-Anteil gewachsen ist (vgl. Karzauninkat 2004b: 90; Roush 2005). Mit "Google-Bombing" werden koordinierte, oft politisch motivierte Aktionen bezeichnet, bei denen durch Verlinkung Seiten in den Ranglisten von Google künstlich nach oben befördert werden. Als in Google bei der Eingabe des Wortes "J ew" an erster Stelle eine antisemitische Website auftauchte, hat eine Vielzahl von Bloggern diese Site verdrängt und den Eintrag "J ew“ der Online-Enzyklopädie Wikipedia an dessen Stelle gesetzt (vgl. Dworschak 2003; Drösser 2004; Karzauninkat 2004b; Livnat 2004).

In einer als Vollerhebung angelegten Befragung deutschsprachiger Suchmaschinen-Anbieter zwischen Oktober 2002 bis Januar 2003 (vgl. Machill/Welp 2003: 83-85) zeigte sich, dass die eher simplen, auch von Laien anwendbaren Formen des Spamming dominierten, nämlich die falsche Charakterisierung von Seiten in den Meta-Tags (durch besonders häufig benutzte Suchwörter, die aber nichts mit dem Inhalt der Seite zu tun haben), die mehrfache Anmeldung von Seiten bei einer Suchmaschine oder die häufige Wiederholung von Wörtern, für die eine Seite optimiert ist. Anspruchsvollere Methoden wie Brückenseiten (mit einer Weiterleitungsfunktion), "Cloaking" (d.h. unterschiedliche Seitenversionen für Robots und Nutzer) und der Aufbau von Linkfarmen tauchten vergleichsweise selten auf.

Zwischen Suchmaschinenanbietern und -optimierern findet eine Art Hase-Igel-Wettlauf statt: Neue Techniken werden rasch durch neue Optimierungsverfahren unterlaufen, was die Suchmaschinen zur Weiterentwicklung zwingt. Suchmaschinen reagieren außerdem durch die Geheimhaltung technischer Details und mit Maßnahmen gegen das Spamming (wie den Ausschluss gespamter Seiten aus dem Index) (vgl. Neue Zürcher Zeitung 2003: 51; Bager 2005a, 2005b).

\section{Interne Manipulation von Suchergebnissen}

Neben der externen Manipulation von Suchmaschinen gibt es auch Formen der internen Manipulation, die von den Suchmaschinen-Anbietern selbst ausgehen. Hier geht es um Treffer, für die Website-Anbieter bezahlen und die nur unzureichend oder gar nicht als Werbung gekennzeichnet sind. Auf diese Praxis aufmerksam gemacht hat im Juli 2001 die Verbraucherschutzorganisation Commercial Alert mit einer Klage bei der Federal Trade Commission (FTC)
(2002). Commercial Alert kritisierte vor allem die unzureichende Kennzeichnung von Werbung außerhalb der Trefferlisten („Paid placement“) und bezahlter Treffer in den Ergebnislisten („Paid inclusion"). Die FTC hat den Suchmaschinen Empfehlungen für die eindeutige Kennzeichnung gegeben, die inzwischen in den großen englischsprachigen Suchmaschinen weitgehend Beachtung finden, wie eine Studie von Consumer Web Watch im Jahr 2004 ergab. Mängel tauchten noch vereinzelt bei der Kennzeichnung von „Paid inclusions“ und bei den Nutzerinformationen ("Disclosure information“) auf (vgl. Sherman 2004; Wouters 2004).

In der erwähnten Anbieterbefragung (vgl. Machill/Welp 2003: 90f.) zeigte sich, dass auch bei deutschsprachigen Suchmaschinen Werbebanner, die thematisch an die Suchanfragen gekoppelt sind (58 \%), und bezahlte Suchresultate, die außerhalb der eigentlichen Trefferliste angezeigt werden (40 $\%)$, als Bezahldienste weite Verbreitung gefunden haben. Beide Formen erscheinen als wenig problematisch, da sie in der Regel durch Gestaltung und Platzierung für die Nutzer gut erkennbar sind. Anders steht es mit erkauften Plätzen in den Trefferlisten selbst, ob mit oder ohne einen garantierten Rangplatz (40\% bzw. $22 \%$ ). Hier dürfte die Verwechslungsgefahr besonders groß sein.

Die befragten Suchmaschinen verwendeten ganz unterschiedliche Wörter zur Markierung bezahlter Treffer. Eindeutig und aus den traditionellen Medien bekannt sind die Hinweise „Anzeige" und "Werbung“. Weniger bekannt sein dürfte, was sich hinter den Bezeichnungen "Sponsored Link“, "Sponsored Listing" oder "Partnerlink" verbirgt. I rreführend sind die Wörter „Empfehlung“ und „Webtipp“, die eher als nutzerorientiertes Qualitätsurteil aufgefasst werden dürften. Einige Anbieter begnügten sich auch mit einer besonderen Schrift und Farbgestaltung, um Bezahltreffer hervorzuheben.

Diverse Studien zeigen, dass viele Online-Nutzer die Praxis bezahlter Treffer nicht kennen, sie oft nicht zwischen bezahlten und nicht-bezahlten Treffern unterscheiden können, sie aber Wert auf eine klare Trennung legen (vgl. Princeton Survey Research Associates 2002: 17; Machill/Welp 2003: 179f.; Marable 2003; Frankfurter Allgemeine Zeitung 2005). So wussten in einer repräsentativen Befragung des Pew Internet \& American Life Project im Mai/J uni 200462 Prozent der Suchmaschinen-Nutzer in den USA nicht Bescheid über diese Praxis. Unter jenen 38 Prozent, welche sie kannten, gaben 47 Prozent an, sie könnten stets erkennen, welche Treffer bezahlt oder gesponsert sind und welche 
nicht. Die Online-Nutzer forderten mehrheitlich eine klare Kennzeichnung. 45 Prozent meinten, sie würden eine Suchmaschine nicht mehr nutzen, falls sie bezahlte Treffer nicht markiert (vgl. Fallows 2005: 16-21).

Suchmaschinen-Marketing gilt als „Motor der Onlinewerbung" (Breuning 2004: 400f.) und ist die Haupterlösquelle vieler Anbieter. Die Stärken des Suchmaschinen-Marketings bestehen darin, dass passend zum Informationsbedürfnis, das der Nutzer durch seine Sucheingabe mitteilt, Werbung geschaltet werden und der Erfolg durch das Anklicken der Links genau kontrolliert werden kann. Google erzielte im Jahr 200395 Prozent seiner Umsätze durch Werbung, die thematisch eingeblendet wird ("AdWords"), und zwar sowohl auf den Ergebnisseiten der Suchmaschine selbst als auch auf Partnersites. $^{2}$ Yahoo hatte einen Werbeanteil am Gesamtumsatz von 82 Prozent, MSN von 29 Prozent (vgl. Bager 2004a; Schwan 2004b; Van Couvering 2004: 7; Ferguson 2005: 42f.). Das Marktforschungsunternehmen eMarketer ermittelte für das Jahr 2004 einen Umsatz von etwa 4 Milliarden USDollar für Suchmaschinen-Marketing in den USA und sagte einen Anstieg für 2005 von 22,5 Prozent voraus (vgl. Delaney 2005a).

Forrester Research (2005) berechnete für Deutschland im Jahr 2004 einen Umsatz im SuchmaschinenMarketing von 165 Millionen Euro und prognostizierte für das Jahr 2010399 Millionen Euro. Ab dem Jahr 2007 werde allerdings europaweit der Marktanteil des Suchmaschinen-Marketing am OnlineWerbemarkt sinken, da das Misstrauen der Nutzer gegenüber bezahlten Treffern wachse, multimediale Werbung („Rich media“) an Bedeutung gewinnen werde und die Preise für "Keyword"-Suche steigen werden. Über die aktuellen Preise für die Belegung der ersten Werbeposition für einen bestimmten Suchbegriff informiert monatlich der deutsche Suchmaschinen-Preisindex (SPIXX), den die Agentur explido WebMarketing führt (explido-webmarketing.de). Preise beziehen sich auf den einzelnen Klick eines Nutzers, ein Abrechnungsverfahren, das ebenfalls Manipulationsmöglichkeiten eröffnet ( $\mathrm{vgl}$. Liedtke 2005).

${ }^{2}$ Allerdings kommt es bei der automatisierten Platzierung der Werbung auch gelegentlich zu absurden Ergebnissen (vgl. Delaney 2005b).

\section{Technische Verzerrung von Suchergebnissen}

Neben der gezielten Manipulation verzerren auch technische Schwächen die Ergebnisse von Suchmaschinen. Qualitätskriterien sind dafür die Relevanz der Treffer und ihre Vollständigkeit, das heißt der Grad der Erfassung der im Internet verfügbaren Dokumente. Suchmaschinen können lediglich auf syntaktischem Niveau operieren, können also weder Seiteninhalte noch Suchanfragen interpretieren (vgl. Kuhlen 1999: 245). Teile des Internets sind für Suchmaschinen technisch nicht oder nur schwer erreichbar (dynamisch generierte Seiten, Multimedia-Angebote, registrierungspflichtige Websites). Außerdem gibt es Kapazitätsgrenzen der Crawler, was dazu führt, dass weniger populäre Bereiche vernachlässigt werden und neue oder aktualisierte Seiten nur mit Verzögerung registriert werden (vgl. Karzauninkat 2004c).

Kritik richtete sich in der letzten Zeit vor allem gegen das Nachrichtenportal Google News, das ohne menschliche Hilfe journalistische Quellen auswertet und Meldungen nach ihrer Relevanz gewichtet (vgl. zum Folgenden: Mrazek 2004; Krüger 2004c; Schink 2005). Zwar sind auch hier die Rankingkriterien geheim, weil aber die redaktionelle Nachrichtenauswahl simuliert werden soll, lässt sich das Ergebnis an journalistischen Standards messen. Als wichtig eingeschätzt werden solche Meldungen, die häufig im Netz vorkommen, was aber nicht unbedingt mit ihrer Relevanz korrelieren muss. Google News bevorzugt außerdem große Medien und Nachrichtenagenturen. Dass knapp die Hälfte aller Nachrichten aus nur fünf Quellen stammt, zeigte eine Auswertung von Digital Deliverance (2004).

Präferiert würden in Google News auch zeitlich aktuellere Beiträge, was dazu führe, dass nicht jene Anbieter, die eine Meldung selbst recherchiert und zunächst exklusiv verbreitet haben, an erster Stelle platziert werden, sondern „Nachzügler“, die sie übernommen haben. Im Vergleich zu Nachrichtensites dauert es oft sehr lange, bis wichtige Meldungen auftauchen. Ein Beispiel: Nachdem der Name des neuen Papstes am 19. April 2005 bekannt gegeben worden war, stand nach rund einer Minute eine Eilmeldung auf der CNN-Website. Das deutschsprachige Google News-Angebot brauchte dagegen fast eine Stunde, bis der Name "Ratzinger" auf der Homepage erschien. Im US-Wahlkampf fiel Beobachtern eine politische "Schräglage“ zugunsten konservativer Positionen auf, was aber - so verteidigte 
sich Google - dem Meinungsbild im Internet entspräche, das von Google News lediglich gespiegelt werde. Ein weiterer Kritikpunkt lautet, dass nicht nur auf seriöse journalistische Quellen verwiesen wird, sondern auch auf PR-Mitteilungen und rechtsextremistische Seiten.

Misstrauen weckt die zurückhaltende Informationspolitik von Google, was das Entfernen von Seiten aus dem Index betrifft. Google differenziert offenbar die Zugänglichkeit von Seiten nach Ländern (etwa in Deutschland, Frankreich und China im Vergleich zur US-Version). Damit hat sich die Suchmaschine den Vorwurf der intransparenten Einflussnahme auf die Suchergebnisse und des voreiligen Nachgebens auf politischen Druck eingehandelt (vgl. z.B. Palm 2002; Zittrain/Edelman 2002; Finkelstein 2003; Jodda 2003; McHugh 2003; Rötzer 2004; Schwan 2004c).

Andererseits werden Google und andere Suchmaschinen auch dafür kritisiert, dass sie den Zugang zu problematischen Seiten ermöglichen (Gewalt, politischer Extremismus, Pornographie), also Seiten nicht filtern oder aus ihrem Index entfernen. Diesen Missstand bestätigen auch die Ergebnisse einer repräsentativen Nutzerbefragung und einer Inhaltsanalyse im Auftrag der Bertelsmann Stiftung. Den Filtern, die den Nutzern angeboten werden, gelingt es nicht zuverlässig, jugendgefährdende Seiten auszusondern (vgl. Machill/Welp 2003: 113-125, 195-205). Überdies soll der von Google eingesetzte Familienfilter SaferSearch auch zahlreiche Seiten ohne pornographische Inhalte blockieren (vgl. Edelman 2003).

\section{Regulierung von Suchmaschinen}

In den letzten beiden Jahren wurde in Deutschland intensiv über die Regulierung von Suchmaschinen diskutiert. Anlass dafür waren Missstände wie die vermutete Meinungsmacht durch die Marktmacht von Google, die Manipulation von Suchergebnissen, die Nichterkennbarkeit bezahlter Treffer, die Zugänglichkeit jugendgefährdender Angebote über Suchmaschinen und der Umgang mit Nutzerdaten. Zur Frage, wie Suchmaschinen juristisch einzuordnen sind, hat die Landesanstalt für Medien Nordrhein-Westfalen (LfM) ein Rechtsgutachten in Auftrag gegeben (vgl. Krempl 2004b).

Mit der Situation auf dem Suchmaschinen-Markt befasste sich im J uni 2004 der Unterausschuss Neue Medien des Deutschen Bundestages. Dabei wurde die Förderung freier Suchmaschinen erwogen (vgl. Heise Online 2004a). Im März 2005 forderte die
Grünen-Fraktion im Bundestag in einer Informationsbroschüre, Alternativangebote zum Marktführer Google zu fördern sowie die Kompetenz der Nutzer zu verbessern, und appellierte an die Suchmaschinen, für Transparenz zu sorgen und Datenschutzvorschriften einzuhalten (vgl. Bundestagsfraktion Bündnis 90/Die Grünen 2005). Ein Eingreifen des Gesetzgebers wurde bislang nicht gefordert.

Dagegen sind mehrere Initiativen zur Selbstregulierung von Suchmaschinen zu verzeichnen: Einen ersten, wenn auch erfolglosen Vorstoß unternahm die Bertelsmann Stiftung. Die Stiftung stellte im Oktober 2003 einen Verhaltenskodex zur Selbstverpflichtung von Suchmaschinen vor, der die Transparenz der Funktionsweise und der Ergebnisse gegenüber den Nutzern, den Schutz vor jugendgefährdenden Inhalten durch Familienfilter, das Entfernen illegaler Seiten sowie eine Zurückhaltung bei der Erfassung von Nutzerdaten forderte. Die Resonanz darauf blieb allerdings sehr gering, sodass auf weitere Schritte (Gütesiegel, Gründung einer freiwilligen Selbstkontrolle) verzichtet wurde (vgl. Bertelsmann Stiftung 2003, 2004).

Dieser Aufgabe nahm sich inzwischen die „Freiwillige Selbstkontrolle Multimedia-Diensteanbieter" (FSM) an, die im Februar 2005 einen Verhaltenskodex für Suchmaschinen vorstellte (vgl. Freiwillige Selbstkontrolle Multimedia-Diensteanbieter 2004). Er enthält neben den oben genannten Punkten auch ein Beschwerdeverfahren und einen Sanktionskatalog. Zu den Gründungsmitgliedern, die sich auf die Einhaltung der Regeln verpflichtet haben, gehören die drei großen Suchmaschinen Google, Yahoo und MSN mit ihren deutschen Ablegern, AOL Deutschland, Lycos Europe, T-Online und t-info (vgl. Freiwillige Selbstkontrolle Multimedia-Diensteanbieter 2005). Weit reichen die Transparenzforderungen, die darin an sie gestellt werden: Für den Nutzer erkennbar sein sollen die Funktionsweise der Suchmaschine und die Kriterien, nach denen Websites ausgeschlossen werden, bezahlte Ergebnisse sollen deutlich gekennzeichnet werden. Die Suchmaschinen haben sich auch verpflichtet, die von der Bundesprüfstelle für jugendgefährdende Medien (BPiM) indizierten Websites nicht zugänglich zu machen. Solche konkreten Hinweise auf problematische Inhalte fehlen allerdings für ausländische Angebote. Außerdem ist die geforderte Filterung technisch nur schwer realisierbar (vgl. Neue Zürcher Zeitung 2005b).

Der Bundesverband Digitale Wirtschaft (BVDW) beschloss im November 2004, künftig ein Zertifikat an seriöse Suchmaschinen-Marketing-Agenturen zu 
vergeben. Damit soll das Spamming von Suchmaschinen eingedämmt werden. Das Zertifikat wird nach Angaben des BVDW von den führenden Suchmaschinen-Vermarktern Espotting, Google und Overture unterstützt. Grundlage für die Aufnahme in die „White List" ist die jährliche Prüfung der Agenturen mit Hilfe eines Kriterienkataloges, den die Mitglieder der BVDW-Arbeitsgruppe SuchmaschinenMarketing ausgearbeitet haben (vgl. BVDW 2005). Für ein Fazit, welche Effekte diese Selbstkontrollen haben, ist es bisher allerdings noch zu früh.

\section{References}

Bager, Jo (2004a): Gerangel an der Bande. Google AdWords - Werbung mit Risiken. In: c't. Nr. 13 v. 14.06.2004, S. 170f.

Bager, Jo (2004b): Jagd auf den Primus. Googles Börsengang birgt viele Risiken. In: c't. Nr. $13 \mathrm{v}$. 14.06.2004, S. 164-169.

Bager, Jo (2005a): Aufsteiger. Websites mit dem Internet Business Promoter suchmaschinengerecht aufbereiten. In: c't. Nr. 9 v. 18.04.2005, S. 158-163.

Bager, Jo (2005b): Turbolift ins Ungewisse. Manipulationsmaschen bei Suchmaschinen. In: c't. Nr. 9 v. 18.04.2005, S. 164-166.

Bauer, Antonie: Google legt spektakulären Börsenstart hin. In: Süddeutsche Zeitung v. 20.8.2004, S. 23.

Bertelsmann Stiftung (2003): Bertelsmann Stiftung legt Verhaltenskodex für Suchmaschinen im Internet vor.

22.10.2003.

"http://www. bertelsmann-

stiftung. de/de/2893. htm" (07.01.2005)

Bertelsmann Stiftung (2004): Suchmaschine crossbot setzt Verhaltenskodex der Bertelsmann Stiftung um. 19.1.02004. "http://www. bertelsmannstiftung.de/de/1013_12680.jsp" (26.11.2004)

Biever, Celeste (2004): Rival engines finally catch up with Google In: New Scientist. Vol. 184, Nr. 2474 v. 20.11.2004, S. 23.

Bleich, Holger/Joerg Heidrich (2004): Mailen mit Google. Gmail unter der Lupe. In: c't. Nr. $10 \mathrm{v}$. 3.5.2004, S. $90 f$.

Breunig, Christian (2004): Online-Werbemarkt in Deutschland 2001 bis 2004. Internet bleibt für die Werbewirtschaft ein Ergänzungsmedium. In: Media Perspektiven. o.J g., H. 8, S. 394-404.

Bundestagsfraktion Bündnis 90/Die Grünen (2005): Suchmaschinen: Das Tor zum Netz. Hintergrund und Information. Nr. 64 v. März 2005. "http://www.gruene-

frakti-

on. de/cms/publikationen/dokbin/63/63265.pdf" (29.03.2005)

BVDW (2005): BVDW zertifiziert SuchmaschinenMarketing-Agenturen.

Pressemitteilung.

08.11.2005.

"http://www.bvdw.org/shared/data/pressclippin

g/2300_001_pm zertifikat $041011 \quad$ Pro-

zent20(3). doc" (03.01.2005)

Computerwoche (2005): Deutsche WebSuchmaschinen nehmen neuen Anlauf. In: Computerwoche. 13.04.2005. "http://www.computerwoche.de" (Datenbankabruf) (18.04.2005)

Delaney, Kevin J. (2005a): Selling Through Search. In: The Wall Street Journal Europe. Nr. $39 \mathrm{v}$. 24.03.2005, S. A9.

Delaney, Kevin J. (2005b): The Trouble With ,Great Deals on Rot'. In: The Wall Street Journal Europe. Nr. 39 v. 24.03.2005, S. A9.

Digital Deliverance (2004): From More Than 4500 Sources, Just a Dozen Account for Most Google News Stories? 02.08.2004. "http://www. digitaldeliverance.com/MT/archives 1000442. html" (07.01.2005)

Drösser, Christoph (2004): Ausgetrickst und zugemüllt. In: Die Zeit. 18.03.2004, S. 39.

Dworschak, Manfred (2003): Sumpfhühner im Anflug. Internet. In: Spiegel Online. 03.11.2003. "http://www.spiegel.de/spiegel/0,1518,272384,0 0.html" (05.11.2003)

Edelman, Benjamin (2003): Empirical Analysis of Google SafeSearch. Last Updated: 10.04.2003. "http://cyber.law. harvard.edu/people/edelman/g oogle-safesearch" (10.04.2003)

eprofessional (2004): Welche Werbewirkung erzielen Textlinks in Suchmaschinen? Eine Studie von Fittkau \& Maaß im Auftrag von eprofessional und in Zusammenarbeit mit AltaVista, Fireball und Lycos. http://www.competencesi-

te.de/marketing.nsf/5A52EEA6497E6C3EC1256E 29006090E1/\$File/studie_werbewirkung_textlink s_eprofessional.pdf (03.01.2005)

Fallows, Deborah (2005): Search Engine Users. Internet searchers are confident, satisfied and trusting - but they are also unaware and naïve. 23.01.2005. Washington, D.C.: Pew Internet \& American Life Project. "http://www.pewinternet.org/pdfs/PIP_Searche ngine_users.pdf" (24.01.2005) 
Fallows, Deborah/Lee Rainie: The popularity and importance of search engines. August 2004. Washington: Pew Internet \& American Life Project.

"http://www. pewinternet.org/pdfs/PIP_Data_Me mo_Searchengines.pdf" (13. 10.2004)

Federal Trade Commission (2002): Commercial Alert Complaint Requesting Investigation of Various Internet Search Engine Companies for Paid Placement and Paid Inclusion Programs. 27.06.2002.

"http://www.ftc.gov/os/closings/staff/commercia lalertattatch. htm" (07.01.2005)

Ferguson, Charles H. (2005): Die netten Jahre sind vorbei. Suchmaschinen. In: Technology Review. Deutsche Ausgabe. H. 2, S. 36-47.

Finkelstein, Seth (2003): Google Censorship - How It Works. 10.03.2003. "http://sethf.com/anticensorware/general/googl e-censorship.php“ (29.03.2005)

Fischermann, Thomas (2004): New Economy, zweiter Versuch. In: Die Zeit. 09.06.2004, S. 21.

Focus Online (2005): Bald Plattform für Videofilmer. Google. In: Focus Online. 25.04.2005. "http://focus.msn.de/hps/fol/newsausgabe/new sausgabe. $h$ tm?id=13926" (27.04.2005)

Forrester Research (2005): European Search Engine Marketing To Grow 65 percent in 2005, Says Forrester Research. In: Tekrati. The Industry Analyst Reporter. 18.03.2005. "http://www. industryanalystreporter.com" (Datenbankabruf) (29.03.2005)

Frankfurter Allgemeine Zeitung (2005): Werbung in Suchmaschinen erlahmt. Internet. In: Frankfurter Allgemeine Zeitung. Nr. 67 v. 21.03.2005, S. 19.

Freiwillige Selbstkontrolle MultimediaDiensteanbieter (2004): Verhaltenssubkodex für Suchmaschinenanbieter des FSM (VK-S). Stand: 21.12.2004.

"http://www. fsm.de/?s=Subkodex+Suchmaschi nenanbi-

eter\&PHPSESSID=b7a49e3ab621bdd5d2a435eb 8e7ee951" (29.04.2005)

Freiwillige Selbstkontrolle MultimediaDiensteanbieter (2005): Suchmaschinenbetreiber gründen Freiwillige Selbstkontrolle unter dem Dach der FSM. 25.2.2005. "http://www.fsm.de/?s=News\&news_id=362\&br owse_page $=2 \& s=$ News\&news $i d=3 \overline{5} 8$ " (29.04.2005)

Glöggler, Michael (2003): Suchmaschinen im Internet. Funktionsweisen, Ranking Methoden, Top
Positionen. Berlin/Heidelberg/New York: Springer.

Golem (2004): Yahoo Deutschland startet lokale Suche mit DasÖrtliche. Lokale Suchergebnisse bei der Suchanfrage. In: Golem. 10.12.2004. "http://www.golem.de/0412/35120.htm/" (07.01.2005)

Heise Online (2004a): Bundestag sorgt sich um Vielfalt im Suchmaschinenmarkt. In: Heise Online.

18.06.2002. "http://www.heise.de/newsticker/meldung/4839 8" (03.01.2005)

Heise Online (2004b): Suchmaschinen-Verein sucht "Wege zum Wissen". In: Heise Online. 23.11.2004.

"http://www.heise.de/newsticker/meldung/5356 8 " (03.01.2005)

Hohensee, Matthias (2004): Geheim wie das CocaCola-Rezept. Suchmaschinen. In: Wirtschaftswoche. 18.03.2004, S. 104-106.

Jodda, Bettina (2003): Vorauseilender Gehorsam als Standard - Google entfernt erneut Seiten aus dem Index, ohne es auf eine gerichtliche Auseinandersetzung ankommen zu lassen. In: Telepolis.

01.09.2003.

"http://www.telepolis.de/deutsch/inhalt/on/1553 5/1. htm/" (01.09.2003)

Karzauninkat, Stefan (2004a): Beziehungsgeflecht der Suchdienste in Deutschland und international.

"http://www.suchfibel. de/5technik/images/such maschinereien_gross.gif" (07.01.2005)

Karzauninkat, Stefan (2004b): Google zugemüllt. Spam überschwemmt die Suchergebnisse. In: c't. Nr. 20 v. 22.9.2004, S. 88-97.

Karzauninkat, Stefan (2004C): Das unsichtbare Netz. 11.11.2004.

"http://www.suchfibel.de/2kunst/das_unsichtbar e_netz.htm" (03.01.2005)

Khopkar, Yashmeet/Amanda Spink/C. Lee Giles/Prital Shah/Sandip Debnath (2003): Search engine personalization: An exploratory study. In: first monday. 8. Jg., H. 7. "http://www. firstmonday.org/issues/issue8_7/kh opkar/index.html" (07.03.2005)

Krempl, Stefan (2004a): Nutch: die freie Suchalternative zu Google. In: Telepolis. 10.06.2004. "http://www. telepolis. de/r4/artikel/17/17592/1. html" (03.01.2005)

Krempl, Stefan (2004b): Suchmaschinen in Deutschland bald nicht mehr jugendfrei? In: Heise Online.

11.5.2004. 
"http://www. heise. de/newsticker/meldung/4725 9" (12.05.2004)

Krüger, Alfred (2004a): Der Kampf um die Inhalte. Privatunternehmen Google als Tor zum Wissen der Menschheit. In: Telepolis. 17.12.2004. "http://www. telepolis. de/r4/artikel/19/19037/1. html (17.12.2004)

Krüger, Alfred (2004b): Hat Google das perfekte Spionagetool? In: Telepolis. 18.10.2004. "http://www. telepolis. de/deutsch/inhalt/te/1860 3/1.htm/" (26.10.2004)

Krüger, Alfred (2004c): Googles Nachrichtenportal in der Kritik. In: Telepolis. 04.10.2004. "http://www. telepolis. de/r4/artikel/18/18463/1. html" (03.01.2005)

Kuhlen, Rainer (1999): Die Konsequenzen von Informationsassistenten. Was bedeutet informationelle Autonomie oder wie kann Vertrauen in elektronische Dienste in offenen Informationsmärkten gesichert werden? Frankfurt a.M.: Suhrkamp.

Laube, Helene (2004): Microsoft drängt in Googles Markt. In: Financial Times Deutschland $v$. 11.11.2004.

http://www.ftd.de/tm/it/1099734364564.htm/ (05.01.2005)

Lee, Felicia R. (2004): The Library, Unbound and Everywhere. In: The New York Times. Articles selected for Süddeutsche Zeitung. 27.12.2004, S. 1 u. 5.

Liedtke, Michael (2005): Jeder Klick kostet der Konkurrenz Bares. Gelinkt. In: Spiegel Online. 15.02.2005.

„http://www.spiegel.de/netzwelt/netzkultur/0,15 18,341817,00. htm/" (15.02.2005)

Livnat, Andrea (2004): Google-Bombing. In: Telepolis.

12.04.2004.

"http://www. telepolis. de/deutsch/inhalt/mein/17 175/1.html" (12.07.2004).

Machill, Marcel (2004): Google ohne Transparenz. Interview geführt von Ulrich Gutmair. In: Netzeitung.

21.05.2004. "http://www.netzeitung. de/voiceofgermany/287 262.htm/" (05.01.2005)

Machill, Marcel/Carsten Welp (Hrsg.) (2003): Wegweiser im Netz. Qualität und Nutzung von Suchmaschinen. Gütersloh: Verlag Bertelsmann Stiftung.

Marable, Leslie (2003): False Oracles: Consumer Reaction to Learning the Truth About How Search Engines Work. Results of an Ethnographic Study. 03.06.2003. Consumer WebWatch. "http://www.consumerwebwatch.org/news/sear chengines/index.htm/" (03.01.2005)

Marr, Mirko (2005): Internetzugang und politische Informiertheit. Zur digitalen Spaltung der Gesellschaft. Konstanz: UVK.

McHugh, Josh (2003): Google vs. Evil. In: Wired. 11. Jg., $\quad H . \quad 1$. "http://www. wired.com/wired/archive/11.01/go ogle_pr.html" (05.01.2004)

Mrazek, Thomas (2004): Google sei mit uns. Nachrichtensuche. In: journalist. H. 3, S. $48 f$.

Neuberger, Christoph (2005): Angebot und Nutzung von Internet-Suchmaschinen. Marktstrategien, Qualitätsaspekte, Regulierungsziele. In: Media Perspektiven. 0.Jg., H. 2, S. 2-13.

Neue Zürcher Zeitung (2003): Das "Googlebombing"-Massaker. Google macht Hochstapler platt. In: Neue Zürcher Zeitung. 13./14.12.2003, S. 51.

Neue Zürcher Zeitung (2004): Das Fotoalbum wandert ins Netz. In: Neue Zürcher Zeitung. 24./25.7.2004, S. 52.

Neue Zürcher Zeitung (2005a): Googeln auf der Festplatte. Suchmaschinen entdecken den Personalcomputer. In: Neue Zürcher Zeitung. Nr. 6 v. 08./09.01.2005, S. 51.

Neue Zürcher Zeitung (2005b): Säuberungsaktion im Internet. Suchmaschinen filtern jugendgefährdende Inhalte. In: Neue Zürcher Zeitung. 04.03.2005.

"http://www.nzz.ch/2005/03/04/em/articleCMW AV.html" (07.03.2005)

Neue Zürcher Zeitung (2005c): Suchroboter als Bücherwurm. Google erschliesst Bibliotheken für die Volltextsuche. In: Neue Zürcher Zeitung. Nr. 71 v. 26./27.03.2005, S. 51.

Neue Zürcher Zeitung (2005d): TV-Programme im Visier der Internet-Suchmaschinen. Neuer Dienst von Yahoo und Google. In: Neue Zürcher Zeitung. Nr. 24 v. 29./30.01.2005, S. 51.

Nielsen NetRatings (2005): Majority of online searchers use multiple search engines, pointing to a fluid competitive landscape, according to Nielsen/Netratings. 28.02.2005. „http://www.nielsennetratings.com/pr/pr_05022 8.pdf" (07.03.2005)

Olsen, Stefanie/Joachim Kaufman (2004): Yahoo stellt Beta von Video-Suchmaschine vor. In: ZDNet.

16.12.2004 "http://www.zdnet.de/news/print this.htm?pid= 39128669-39001023c" (19.12.2004)

Palm, Goedart (2002): Die Welt ist fast alles, was Google ist. In: Telepolis v. 28.03.2002. 
"http://www.heise.de/tp/r4/artikel/12/12187/1.h tm/" (07.01.2005)

Patalong, Frank (2004a): Harte Zeiten für Google. Suchdienste. In: Spiegel Online v. 18.11.2004. "http://www. spiegel.de/netzwelt/technologie/0, 1518,328480,00. htm/" (18.11.2004)

Patalong, Frank (2004b): G-Mail statt E-Mail. Google greift an. In: Spiegel Online. 01.04.2004. "http://www.spiegel.de/netzwelt/technologie/0, 1518,293502,00. htm/" (01.04.2004)

Princeton Survey Research Associates (2002): A Matter of Trust: What Users Want From Web Sites. Results of a National Survey of Internet Users for Consumer Webwatch. Januar 2002. "http://www.consumerwebwatch.com/news/rep ort1.pdf" (07.01.2003)

Rötzer, Florian (2004): Google-Anzeige für das Nicholas Berg-Video. In: Telepolis. 21.05.2004. "http://www. telepolis. de/deutsch/inhalt/te/1747 0/1.html“ (24.05.2004)

Roush, Wade (2005): Amazon für alle. In: Technology Review. Deutsche Ausgabe. H. 2, S. 16-18.

Sander-Beuer, Wolfgang (2005): Schürfrechte im Informationszeitalter Google hin, Microsoft her das Internet braucht eine freie Suchkultur. In: Technology Review. Deutsche Ausgabe. H. 2, S. 29.

Schink, Peter (2005): Google News entfernt nationalistische Zeitung. In: Netzeitung. 24.03.2005. "http://www.netzeitung. de/internet/331187.htm I" (29.03.2005)

Schwan, Ben (2004a): Blogger.com internationalisiert. In: Netzeitung. 16.11.2004. "http://www.netzeitung. de/internet/313325.htm I" (26.11.2004)

Schwan, Ben (2004b): Google will geschickter werben. In: Netzeitung. 11.05.2004. "http://www.netzeitung. de/internet/285996.htm I" (13.05.2004)

Schwan, Ben (2004b): Kritik an Googles ChinaPolitik. In: Netzeitung. 01.12.2004. "http://www.netzeitung. de/internet/315301.htm I" (07.12.2004)

Seekport (2004): Presseinformation: 60 Prozent der deutschen Internet-Nutzer beklagen Infomüll in suchmaschinen.

11.11.2004. "http://www.seekport.de/presse/PM Forsa.pdf" (16.11.2004).

Seifert, Heribert (2003): Mehr Recycling als Recherchen. Zum Zustand des investigativen Journalismus. In: Neue Zürcher Zeitung. 10./11.5.2003, S. 50.
Sherman, Chris (2004): Rating Search Engine Disclosure Practices. In: Search Engine Watch. 24.11.2004.

"http://searchenginewatch.com/reports/article.p $h p / 34711$ 3439401" (03.01.2005)

Spiegel Online (2004): Googles Probleme mit dem Briefgeheimnis. Datenschützer besorgt. In: Spiegel Online. 08.04.2004. "http://www.spiegel.de/netzwelt/politik/0,1518, 294559, 00. html" (08.04.2004)

Spiegel Online (2005): Google und Yahoo durchsuchen TV-Angebote. Suchtechnik. In: Spiegel Online.

25.01.2005.

"http://www.spiegel.de/netzwelt/technoogie/0,1 $518,338515,00 . \mathrm{html}$ " (25.01.2005)

sueddeutsche.de (2005): "Googel local" geplant. Neue Suchfunktion. In: sueddeutsche.de. 24.03.2005.

"http://www.sueddeutsche.de/wirtschaft/artikel/ 51/50001/print.htm/“ (29.03.2005)

Sullivan, Danny (2004): Who Powers Whom? Search Providers Chart. In: Search Engine Watch. 23.07.2004.

"http://searchenginewatch.com/reports/article.p hp/34701_2156401" (03.01.2005)

Van Couvering, Elizabeth (2004): New Media? The Political Economy of Internet Search Engines. A paper presented to The Communication Technology Section, 2004 Conference of the International Association of Media \& Communications Research (IAMCR). Porto Alegre, Brazil, 25.30.7.1994.

van Eimeren, Birgit/Heinz Gerhard/Beate Frees (2003): Internetverbreitung in Deutschland: Unerwartet hoher Zuwachs. ARD/ZDF-OnlineStudie 2003. In: Media Perspektiven. 0.J g., H. 8, S. 338-358.

van Eimeren, Birgit/Heinz Gerhard/Beate Frees (2004): Internetverbreitung in Deutschland: Potenzial vorerst ausgeschöpft? ARD/ZDF-OnlineStudie 2004. In: Media Perspektiven. 0.J g., H. 8, S. $250-370$.

Virtel, Martin (2005): Google erhöht Investitionsbudget für 2005 auf 500 Mio. Dollar. In: Financial Times Deutschland. 04.04.2005. "http://www.ftd. de/tm/tk/1780.htm/" (06.04.2005)

Wegner, Jochen (2005): Bauer Poppe und die Googleisierung. In: jonet. 12.01.2005. "http://jochen.jonet.org/modules.php?name=Ne ws\&file=article\&sid=119" (19.01.2005)

Wiegandt, Kai Martin (2004): Googles Gegner. Frankreich will Bibliotheken digitalisieren - und 
dem US-Unternehmen Paroli bieten. In: Süddeutsche Zeitung. Nr. 47 v. 26./27.02.2005, S. 15

Winkler, Hubert (2002): Suchmaschinen. Metamedien im Internet? In: Navigationen. Siegener Beiträge zur Medien- und Kulturwissenschaft. 2. Jg., H. 2, S. 33-42.

Winterbauer, Stefan (2003): Das Googlepol. Die Medienmacht einer Internet-Suchmaschine. In: Süddeutsche Zeitung. 19./20.7.2003, S. 18.

Wouters, Jørgen J. (2004): Searching for Disclosure: How Search Engines Alert Consumers to the Presence of Advertising in Search Results. A Report for Consumer Web Watch. 08.11.2004. "http://www.consumerwebwatch.org/news/paid search/finalreport.pdf" (07.01.2005)
Zittrain, Jonathan/Benjamin Edelman (2002): Localized Google search result exclusions. Statement of issues and call for data. Last Updated: 26.10.2002.

"http://cyber.law.harvard.edu/filtering/google/" (07.01.2005) 